\title{
Knowledge about HPV and Screening of Cervical Cancer among Women from the Metropolitan Region of Natal, Brazil
}

\author{
Érika Galvão Lima, ${ }^{1}$ Diego Breno Soares de Lima, ${ }^{1}$ Cleine Aglacy Nunes Miranda, ${ }^{1}$ \\ Valeska Santana de Sena Pereira, ${ }^{1}$ Jenner Chrystian Veríssimo de Azevedo, ${ }^{2}$ \\ Josélio Maria Galvão de Araújo, ${ }^{3}$ Thales Allyrio Araújo de Medeiros Fernandes, ${ }^{4}$ \\ Paulo Roberto Medeiros de Azevedo, ${ }^{5}$ and José Veríssimo Fernandes ${ }^{3}$ \\ ${ }^{1}$ Post-Graduate Program in Biological Sciences, Biosciences Center, Federal University of Rio Grande do Norte, \\ Avenida Sen. Salgado Filho s/n, Campus Universitário, Lagoa Nova, 59072-970 Natal, RN, Brazil \\ ${ }^{2}$ Pediatric Hospital, Federal University of Rio Grande do Norte, Rua Gal. Cordeiro de Farias, s/n, Petrópolis, \\ 59012-570 Natal, RN, Brazil \\ ${ }^{3}$ Department of Microbiology and Parasitology, Federal University of Rio Grande do Norte, Avenida Sen. Salgado Filho s/n, \\ Campus Universitário, Lagoa Nova, 59072-970 Natal, RN, Brazil \\ ${ }^{4}$ Department of Biomedical Sciences, University of Rio Grande do Norte State, Rua Atirador Miguel Antônio da Silva Neto, \\ $s / n$, Aeroporto, Mossoró, RN, Brazil \\ ${ }^{5}$ Department of Statistics, Center of Exact Sciences and Earth, Federal University of Rio Grande do Norte, \\ Avenida Sen. Salgado Filho s/n, Campus Universitário, Lagoa Nova, 59078-970 Natal, RN, Brazil
}

Correspondence should be addressed to José Veríssimo Fernandes; veris@cb.ufrn.br

Received 18 February 2013; Accepted 13 March 2013

Academic Editors: M. Friedrich, N. A. Ginsberg, C. Iavazzo, S. Palomba, and K. Yang

Copyright ( 2013 Érika Galvão Lima et al. This is an open access article distributed under the Creative Commons Attribution License, which permits unrestricted use, distribution, and reproduction in any medium, provided the original work is properly cited.

\begin{abstract}
Objective. The purpose of this study was to assess the knowledge level about HPV and screening of cervical cancer in women from the metropolitan region of Natal, Brazil. Materials and Methods. A descriptive cross-sectional study involving sexually active women was conducted. The participants were submitted to a face-to-face interview, using a structured questionnaire that permitted the quantification of data and opinions of the respondents. Results. Most participants (70.9\%) had poor knowledge about HPV and also the Pap test (53.0\%). The high level of knowledge about HPV was associated with age, education, marital status, household income, and pregnancy, while the high level of knowledge about the Pap test proved to be associated only with education and household income. Conclusion. The results highlight the need for performing educational campaigns emphasizing the role of HPV in the etiology of cervical lesions of different degrees, including cervical cancer, as well as the importance of having a Pap test regularly to prevent these diseases.
\end{abstract}

\section{Introduction}

Cervical cancer is the third most common cancer affecting women, after nonmelanoma skin cancer and breast cancer, and is the third leading cause of death by cancer among women worldwide, with higher incidence in developing countries [1,2]. It is estimated that about 500,000 new cases are reported every year, with approximately 230,000 deaths worldwide. In Brazil, the crude incidence rates per 100,000 women, estimated for the year 2012, were 17 for the country and 14 for the Rio Grande do Norte State. The incidence of the disease starts from the age of 20 and the risk gradually increases with age, reaching its peak generally at age 50 to 60 [3].

The natural history of cervical cancer reveals that, despite its high incidence, this malignant neoplasm stands out among those with the greatest potential for prevention and cure in view of their infectious nature [4]. Cervical cytology is one of the most efficient methods for the screening of cervical cancer and is the most commonly used method worldwide 
[5]. Despite its benefits, many women in developing countries have never been screened or are not screened at regular intervals, so that this screening program does not have the desired impact on women's health [6]. Reasons for the lack of effectiveness of these screening programs in developing countries include low screening coverage and participation rates among women, lack of quality control, poor ability of the health care system to offer proper followup and access to colposcopy, and inappropriate final diagnosis and treatment [7].

There is no doubt that when women face participation decisions, it is ideal for them to have a good understanding and perception of the risk of acquiring cervical cancer and the benefits of preventing it [7]. In Brazil, the coverage of the cytopathology exam has not still reached the desired indices. It is estimated that approximately $40 \%$ of Brazilian women have never undergone the procedure [3]. This is due to several factors, including the difficulty of access to health services, poor knowledge about the Pap smear, and the lack of awareness of the benefits that this exam brings to women's health [8].

Human papillomavirus (HPV) is one of the most common causes of sexually transmitted disease in both men and women around the world, especially in developing countries. The prevalence of asymptomatic infection varies from 2 to $44 \%$, depending on the population and studied region [9]. Most HPV infections are transient and some studies show that the majority of sexually active individuals are exposed to and acquire infection from this virus at some phase in their lives.

HPV infection is more prevalent in young adults, at the beginning of their sexual activity, with a subsequent decline in the prevalence rate with increasing age, likely as a result of the development of an immune response against the virus and reduction of sexual activity $[10,11]$. Currently, it is known with certainty that HPV is the etiologic agent of virtually all cases of cervical cancer and is responsible for a high proportion of preinvasive cervical lesions as well as genital warts and other nongenital cancers [12].

Today, more than 150 different HPV types have been cataloged and about 40 can infect the epithelial lining of the anogenital tract and other mucosal areas of the human body. Based on their association with cervical cancer and precursor lesions, HPVs are classified as high-risk (HR-HPV) and lowrisk (LR-HPV) oncogenic types. LR-HPV types, such as HPV 6 and 11, can cause common genital warts or benign hyperproliferative lesions with very limited tendency to malignant progression, while infection with HR-HPV types, highlighting HPV 16 and 18, is associated with the occurrence of premalignant and malignant cervical lesions [13]. HR-HPV types are also associated with many penile, vulvar, anal, and head and neck carcinomas, and contribute to over $40 \%$ of oral cancers [14].

The recognition of the central role of HPV in the etiology of cervical cancer has dramatically changed the vision of how to prevent this cancer. Among the strategies, introduction of HPV testing for primary screening or as an adjunct test and the introduction of HPV vaccines to prevent HPV infection are being evaluated in different settings around the world [15].
Indeed, these advances are available in many countries that have approved the introduction of the HPV vaccine. However, gaps in knowledge about the causal role of HPV in cervical cancer and the benefits of preventing HPV infection may hamper the successful introduction of the technologies. The greater the women's knowledge of HPV and its role in the development of cervical cancer, the greater will be the adherence to preventive measures.

The objective of this study was to evaluate the knowledge level about HPV and the Pap smear screening among high school students and women treated in the health units of the metropolitan region of Natal, Brazil.

\section{Materials and Methods}

2.1. Study Design and Participants. This was a descriptive cross-sectional study carried out during the period of January 2009 to December 2011 involving sexually active women of the metropolitan region of Natal, capital of Rio Grande do Norte State, Northeastern Brazil. We chose basic health units and public schools, distributed throughout all the 10 municipalities that make up the metropolitan region of Natal, to participate in the research.

The interviewers approached potential female participants among the high school students of the selected schools and among women attending basic health units during the study period. In the schools, some classes were randomly chosen and the students were invited by interviewers to participate in the study. In the health units, women who sought by spontaneous demand the screening test for cervical cancer were invited to participate in the study. After agreeing to participate in the study, the women aged 18 years old or older signed an informed consent before survey administration. Those under 18 years had their participation authorized by parents or guardians. A total of 706 participants, aged 14 to 59 years, were included in the study. The only inclusion criteria were to be of female gender, to have already initiated sexual activity, and to agree to participate in the research.

This study was approved by the Research Ethics Committee of Federal University of Rio Grande do Norte.

2.2. Data Collection. All interviews were conducted faceto-face by trained enumerators using a paper structured questionnaire, containing 25 questions including 15 questions about age, ethnicity, marital status, education, household income, sexual activity, and reproductive health history. There were six questions regarding Pap smear knowledge, including such questions as (1) Have you previously heard about this exam? (2) What is the purpose of this exam? and (3) What is the frequency with which the exam must be done? Other questions concerned the level of awareness of the respondents on the advantages and benefits of the examination for the health of women. The remaining four questions were related to HPV knowledge, including questions about the potential consequences of HPV and the awareness of the signs and symptoms, and mode of transmission. All the Pap test and HPV knowledge questions were accompanied by multiple-choice answers, one of which was considered the correct answer to the question. The interviewers were 
instructed to recite all multiple-choice answers to the participants and then ask for the answer to each question.

2.3. Data Analysis. We defined the level of knowledge according to the number of correct responses. Knowledge about Pap screening was categorized as high, medium, or low based on the six Pap-related questions in the survey. A participant with a high level of knowledge answered at least five of the six Pap knowledge questions correctly; participants with a medium level of knowledge answered three or four of the six questions correctly, and participants with a low level of knowledge answered none or at most two of the questions correctly. Women who responded correctly to all four HPVrelated questions were categorized as having a high level of knowledge on the virus, those who answered two or three questions correctly were categorized as having a medium level of knowledge, and the women who responded correctly to only one or none of the HPV-related questions were categorized as having a low knowledge level.

The participant ethnicity was defined based on selfreports according to the criterion of the Instituto Brasileiro de Geografia e Estatística (IBGE), which classifies ethnicity into five categories: white, black, mulatto, Asian, and "native," which were combined into a nonwhite category. We considered as having low income those participants who reported a household income of up to the minimum wage $(678.00$ reais/month), equivalent to US\$ 320.00; as middle income, those with a household income between one and four times the minimum wage, and as high-income, women who reported a household income higher than four times the minimum wage. For the purposes of analysis, women with high Pap screening knowledge were compared against those with low and medium knowledge. Similarly, women with a high level of knowledge about HPV were compared with those who demonstrated a low level of knowledge on the virus.

2.4. Statistical Analyses. To verify the association between the variables studied and the knowledge level for HPV and Pap test, we used the odds ratio (OR) and its 95\% confidence intervals (CIs), according to the univariate regression model, using the software SPSS, version 17.0, and PEPI. Statistical significance within each group was evaluated by Pearson's $\chi^{2}$ test. $P$ value $\leq 0.05$ was considered statistically significant.

\section{Results}

3.1. Profile of the Participants. The profile of the segment of the population studied is presented in Table 1 . The age range of respondents was $14-59$ years with a mean of 30.4 years and $\mathrm{SD}$ of \pm 11.5 years.

Most (51.8\%) of the studied population consisted of young women aged 14 to 28 years, $52.3 \%$ were of nonwhite ethnicity, and $64.2 \%$ were either married or living in a stable relationship with her partner. Regarding the level of education, $37.1 \%$ reported that they had only elementary school level or less, $52.4 \%$ were attending high school or had completed high school, and only $10.5 \%$ reported being enrolled in or having completed some type of education above high school. The vast majority (78.9\%) of the women interviewed

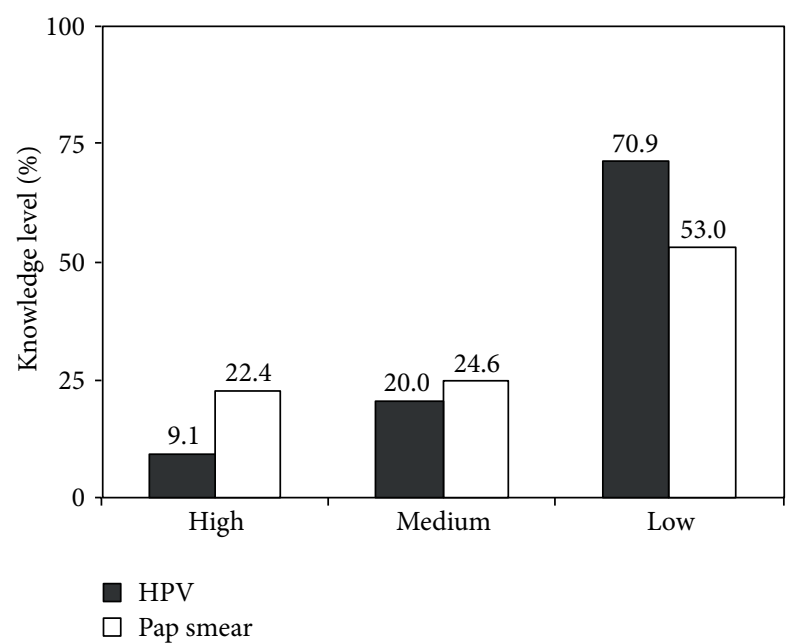

Figure 1: Distribution of knowledge level related to Pap test and HPV. A high proportion of the women had low knowledge related to both Pap test and HPV.

reported a low income (household income of up to $\mathrm{R} \$$ 678.00 reais/month), equivalent to US\$320.00. Most of them (52.8\%) had never had a pregnancy, and among those who had been pregnant, the majority $(81.0 \%)$ had between one and three children.

3.2. Pap Test Knowledge. Participants of this study showed a low level of knowledge about Pap test screening. When asked if they knew what a Pap test was, $53.0 \%$ of the respondents indicated that they knew about the exam and were able to say what it means, and $48.0 \%$ of them were able to provide the correct frequency with which the test should be done and to demonstrate awareness of the advantages and benefits of performing the procedure regularly. According to the level of knowledge presented, participants were categorized as having low, medium, or high level of knowledge about the Pap test screening, based on the correct number of responses. Of the women surveyed, $22.4 \%$ exhibited a high level of knowledge about the Pap test, $24.6 \%$ had a medium knowledge level, and the majority (53.0\%) were categorized as having a low level of knowledge about the exam (Figure 1).

Table 2 shows the Pearson's Chi-square $P$ values association between the considered variables and Pap knowledge. We found that the high knowledge level of the Pap test was associated with level of education $(P<0.001)$ and household income $(P<0.001)$, while the middle-level knowledge of this exam was associated with marital status $(P<0.001)$ and pregnancy $(P<0.001)$. Association between the Pap test knowledge level according to the considered variables and odds ratio (OR) can be seen in Table 4 .

Having or not having the high-level knowledge of the Pap test was considered the dependent variable, and independent variables were those identified as significant in Table 2. We found that having a high school education or above versus elementary school education or less $(P<0.001)$ and having a middle or high household income versus low household income $(P<0.001)$ were related to an increase of the Pap 
TABLE 1: Demographic and behavioral characteristics of survey respondents in the metropolitan region of Natal, Brazil.

\begin{tabular}{|c|c|c|}
\hline Característica & $N$ & $\%$ \\
\hline \multicolumn{3}{|l|}{ Age (years) } \\
\hline $14-28$ & 366 & 51.8 \\
\hline $29-38$ & 180 & 25.5 \\
\hline $39-48$ & 92 & 13.0 \\
\hline $49-59$ & 68 & 9.6 \\
\hline \multicolumn{3}{|l|}{ Ethnicity } \\
\hline White & 337 & 47.7 \\
\hline Nonwhite & 369 & 52.3 \\
\hline \multicolumn{3}{|l|}{ Education } \\
\hline Elementary school or less & 262 & 37.1 \\
\hline Attending high school or high school & 370 & 52.4 \\
\hline Above high school & 74 & 10.5 \\
\hline \multicolumn{3}{|l|}{ Marital status } \\
\hline Single & 253 & 35.8 \\
\hline Married or accompanied & 453 & 64.2 \\
\hline \multicolumn{3}{|l|}{ Household income } \\
\hline Low income & 557 & 78.9 \\
\hline Middle or high income & 121 & 17.1 \\
\hline High income & 28 & 4.0 \\
\hline \multicolumn{3}{|l|}{ Pregnancy } \\
\hline No & 375 & 53.1 \\
\hline Yes & 331 & 46.9 \\
\hline \multicolumn{3}{|l|}{ Number of pregnancies } \\
\hline 0 & 375 & 52.8 \\
\hline $1-3$ & 268 & 38.0 \\
\hline$\geq 4$ & 63 & 8.9 \\
\hline
\end{tabular}

test knowledge score. The odds ratio of having a high Pap knowledge score was 10.2 times greater among women with high school or above, compared with those with elementary school or less (95\% CI 5.534-8.825), and 2.68 times greater for the women with a middle or high household income, compared with those with a low household income (95\% CI 1.810-3.987).

With regard to the level of knowledge of the Pap test, among the 706 women surveyed, 96.2\% had heard about it, but only $47.0 \%$ had medium or high knowledge related to the procedure. The main source of information about the Pap test was health care staff, mentioned by $45.2 \%$ of the respondents, followed by friends and family with $22.5 \%$. We found that $87.4 \%$ of the interviewed women reported that they had had at least one Pap test during their lifetime; $69.4 \%$ had had the procedure done with the frequency of at least once every three years, as recommended by the Ministry of Health; $18 \%$ said they had not had the test in the last 3 years, and $12.6 \%$ reported that they had never had the test throughout their life. When asked about the reasons for not having the procedure, the responses most commonly mentioned by these women were that they felt that nothing was wrong
(28.6\%), neglect (23.4\%), had fear of pain (12.2\%), and felt ashamed of exposing their intimacy (6.8\%).

3.3. HPV Knowledge. Participants of this study presented a very low level of knowledge related to HPV. Only a minority of the women interviewed were able to quote correctly the transmission mode of the HPV and identify the signs and symptoms of the infection caused by this virus, as well as consciously recognize the relationship between the genital HPV infection and the occurrence of cervical lesions including cancer. A significant portion of respondents (64.2\%) said they had heard about HPV, but a much smaller proportion (29.1\%) demonstrated having some knowledge about the virus. Only $20.0 \%$ of the women respondents knew that HPV is a sexually transmitted agent, and a very small percentage (9.1\%) of participants were able to establish the correlation between HPV and the occurrence of anogenital warts and of premalignant cervical lesions and cervical cancer.

Thus, when subjects were analyzed according to the level of knowledge presented about HPV, it was found that most $(70.9 \%)$ of the participants were categorized as having low knowledge about the virus; $20.0 \%$ exhibited a medium knowledge level, and only $9.1 \%$ were categorized as having a high level of knowledge about HPV (Figure 1). Table 3 shows the analysis by means of Pearson's Chi-square to verify the existence of the association between knowledge of HPV and the variables studied. $P$ values confirmed the existence of association between knowledge level of the virus with age, $(P<0.001)$, education $(P<0.001)$, household income $(P<$ $0.001)$, pregnancy $(P<0.001)$, and marital status $(P=$ 0.006).

Association between HPV knowledge level according to the considered variables and odds ratio (OR) can be seen in Table 5. Having a high knowledge level or not about HPV was considered the dependent variable, while independent variables were those identified as significant in Table 3 . We found an increase of the HPV knowledge score in the women aged between 39 and 48 years $(P<0.001)$ and 49 to 59 years $(P=0.001)$ versus those aged between 14 and 28 years; in those having high school education or above versus elementary school education or less $(P<0.001)$; in the married or accompanied versus single $(P=0.003)$; and in those with middle or high household income versus low household income $(P<0.001)$. The odds ratio of having a high HPV knowledge score was 4.50 times greater in women aged $39-48$ years (95\% CI 2.289-8.859) and 3.71 times greater in those aged between 49 and 59 years $(95 \%$ CI 1.718-8.001), compared with those aged between 14 and 28 years. Furthermore, we observed values of the odds ratio of 43.16 times greater in women with high school or above, compared with those with elementary school or less $(95 \%$ CI 5.534-8.825); of 1.92 times greater in women married or accompanied, compared with single women (95\% CI 1.0563.507); 3.11 times greater in those with a middle or high household income compared to those with a low household income (95\% CI 1.824-5.305); and 3.81 times greater in women who had at least one pregnancy, compared with those who had never been pregnant (95\% CI 2.116-6.844). 
TABLE 2: Frequency, distribution, and univariate associations of characteristics in relation to Pap knowledge level.

\begin{tabular}{|c|c|c|c|c|c|}
\hline \multirow{2}{*}{ Characteristic } & \multirow{2}{*}{$N=706$} & \multicolumn{3}{|c|}{ Pap test knowledge score } & \multirow{2}{*}{$P$} \\
\hline & & Low & Medium & High & \\
\hline Age (years) & & & & & 0.237 \\
\hline $14-28$ & 366 & $207(56.6)$ & $78(21.3)$ & $81(22.1)$ & \\
\hline $29-38$ & 180 & $93(51.7)$ & $47(26.1)$ & $40(22.2)$ & \\
\hline $39-48$ & 92 & $39(42.4)$ & $29(31.5)$ & $24(26.1)$ & \\
\hline $49-59$ & 68 & $35(51.5)$ & $20(29.4)$ & $13(19.1)$ & \\
\hline Ethnicity & & & & & 0.484 \\
\hline White & 337 & $173(51.3)$ & $82(24.3)$ & $82(24.3)$ & \\
\hline Nonwhite & 369 & $201(54.5)$ & $92(24.9)$ & $76(20.6)$ & \\
\hline Education & & & & & 0.000 \\
\hline Elementary school or less & 262 & $171(65.3)$ & $79(30.2)$ & $12(4.6)$ & \\
\hline High school and above & 444 & $203(45.7)$ & $95(21.4)$ & $146(32.9)$ & \\
\hline Marital status & & & & & 0.000 \\
\hline Single & 253 & $157(62.0)$ & $37(14.6)$ & $59(23.3)$ & \\
\hline Married or accompanied & 453 & $217(47.9)$ & $137(30.2)$ & $99(21.8)$ & \\
\hline Household income & & & & & 0.000 \\
\hline Low income & 557 & $317(56.9)$ & $138(24.8)$ & $102(18.3)$ & \\
\hline Middle or high income & 149 & $57(38.3)$ & $36(24.2)$ & $56(37.6)$ & \\
\hline Pregnancy & & & & & 0.000 \\
\hline No & 375 & $230(61.3)$ & $60(16.0)$ & $85(22.7)$ & \\
\hline Yes & 331 & $144(43.5)$ & $114(34.4)$ & $73(22.1)$ & \\
\hline
\end{tabular}

TABLE 3: Frequency, distribution, and univariate associations of characteristics in relation to HPV knowledge level.

\begin{tabular}{|c|c|c|c|c|c|}
\hline \multirow{2}{*}{ Characteristic } & \multirow{2}{*}{$N=706$} & \multicolumn{3}{|c|}{ HPV knowledge score } & \multirow{2}{*}{$P$} \\
\hline & & Low & Medium & High & \\
\hline Age (years) & & & & & 0.000 \\
\hline $14-28$ & 366 & $260(71.0)$ & $86(23.5)$ & $20(5.5)$ & \\
\hline $29-38$ & 180 & $125(69.4)$ & $42(23.3)$ & $13(7.2)$ & \\
\hline $39-48$ & 92 & $62(67.4)$ & $11(12.0)$ & $19(20.7)$ & \\
\hline $49-59$ & 68 & $54(79.4)$ & $2(2.9)$ & $12(17.6)$ & \\
\hline Ethnicity & & & & & 0.061 \\
\hline White & 337 & $225(66.8)$ & $78(23.1)$ & $34(10.1)$ & \\
\hline Nonwhite & 369 & $276(74.8)$ & $63(17.1)$ & $30(8.1)$ & \\
\hline Education & & & & & 0.000 \\
\hline Elementary school or less & 262 & $237(90.5)$ & $24(9.2)$ & $1(0.4)$ & \\
\hline High school or above & 444 & $264(59.5)$ & $117(26.4)$ & $63(14.2)$ & \\
\hline Marital status & & & & & 0.006 \\
\hline Single & 253 & $174(68.8)$ & $64(25.3)$ & $15(5.9)$ & \\
\hline Married or accompanied & 453 & $327(72.2)$ & $77(17.0)$ & $49(10.8)$ & \\
\hline Household income & & & & & 0.000 \\
\hline Low income & 557 & $424(76.1)$ & $96(17.2)$ & $37(6.6)$ & \\
\hline Middle or high income & 149 & $77(51.7)$ & $45(30.2)$ & $27(18.1)$ & \\
\hline Pregnancy & & & & & 0.000 \\
\hline No & 375 & $263(70.1)$ & $96(25.6)$ & $16(4.3)$ & \\
\hline Yes & 331 & $238(71.9)$ & $45(13.6)$ & $48(14.5)$ & \\
\hline
\end{tabular}


TABLE 4: Distribution of respondents by Pap knowledge level according to the considered variables and associated odds ratio (OR) obtained by univariate logistic regression model.

\begin{tabular}{|c|c|c|c|c|c|c|}
\hline \multirow{2}{*}{ Characteristic } & \multirow{2}{*}{$N=706$} & \multicolumn{4}{|c|}{ Pap knowledge score } & \multirow{2}{*}{$P$} \\
\hline & & Low or medium & High & OR & $\mathrm{CI}$ & \\
\hline Education & & & & & & 0.000 \\
\hline Elementary school or less & 262 & 250 & $12(4.6)$ & 1.00 & [Reference] & \\
\hline High school or above & 444 & 298 & $146(32.9)$ & 10.20 & {$[5.534-8.825]$} & \\
\hline Household income & & & & & & 0.000 \\
\hline Low income & 557 & 455 & $102(18.3)$ & 1.00 & [Reference] & \\
\hline Middle or high income & 149 & 93 & $56(37.6)$ & 2.68 & {$[1.810-3.987]$} & \\
\hline Marital status & & & & & & 0.920 \\
\hline Single & 253 & 194 & $59(23.3)$ & 1.00 & [Reference] & \\
\hline Married or accompanied & 453 & 354 & $99(21.8)$ & 0.92 & {$[0.637-1.327]$} & \\
\hline Pregnancy & & & & & & 0.965 \\
\hline No & 375 & 290 & $85(22.7)$ & 1.00 & [Reference] & \\
\hline Yes & 331 & 258 & $73(22.1)$ & 0.97 & {$[0.677-1.377]$} & \\
\hline
\end{tabular}

TABLE 5: Distribution of respondents by HPV knowledge level according to the considered variables and associated odds ratio (OR) obtained by univariate logistic regression model.

\begin{tabular}{|c|c|c|c|c|c|c|}
\hline \multirow{2}{*}{ Characteristic } & \multicolumn{5}{|c|}{ Number of percentage of women (\%) HPV knowledge score } & \multirow{2}{*}{$P$} \\
\hline & $N=706$ & Low or medium & High & OR & $\mathrm{CI}$ & \\
\hline \multicolumn{7}{|l|}{ Age (years) } \\
\hline $14-28$ & 366 & 346 & $20(5.5)$ & 1.00 & [Reference] & \\
\hline $29-38$ & 180 & 167 & $13(7.2)$ & 1.35 & {$[0.654-2.773]$} & 0.419 \\
\hline $39-48$ & 92 & 73 & $19(20.6)$ & 4.50 & {$[2.289-8.859]$} & 0.000 \\
\hline $49-59$ & 68 & 56 & $12(17.6)$ & 3.71 & {$[1.718-8.001]$} & 0.001 \\
\hline \multicolumn{7}{|l|}{ Education } \\
\hline Elementary school or less & 262 & 261 & $1(0.4)$ & 1.00 & [Reference] & \\
\hline High school or above & 444 & 381 & $63(14.2)$ & 43.16 & {$[5.948-3.130]$} & 0.000 \\
\hline \multicolumn{7}{|l|}{ Marital status } \\
\hline Single & 253 & 238 & $15(5.9)$ & 1.00 & [Reference] & \\
\hline Married or accompanied & 453 & 404 & $49(10.8)$ & 1.92 & {$[1.056-3.507]$} & 0.033 \\
\hline \multicolumn{7}{|l|}{ Household income } \\
\hline Low income & 557 & 520 & $37(6.6)$ & 1.00 & [Reference] & \\
\hline Middle or high income & 149 & 122 & $27(18.1)$ & 3.11 & {$[1.824-5.305]$} & 0.000 \\
\hline \multicolumn{7}{|l|}{ Pregnancy } \\
\hline No & 375 & 359 & $16(2.3)$ & 1.00 & [Reference] & \\
\hline Yes & 331 & 283 & $48(14.5)$ & 3.81 & {$[2.116-6.844]$} & 0.000 \\
\hline
\end{tabular}

\section{Discussion}

Cervical cancer screening is a diagnosis resource of fundamental importance to women's health in that it allows early detection of the precursor lesions and the therapeutic intervention, before they progress to the malignant form. Because of its infectious nature, cervical cancer has a high potential for prevention, which can be put into practice by avoiding risky behaviors for acquisition of the HPV infection, as well as through the use of available vaccines. Thus, knowledge about the role of HPV in the development of the disease becomes of paramount importance for improving the degree of awareness of women to adopt measures to reduce the risks of infection by this virus, as well as to have early diagnosis of the existing infections. In this study we evaluated the degree of knowledge about HPV and the Pap test in a segment of the female population of the metropolitan region in Natal, Brazil.

According to the official data, the metropolitan region of Natal consists of 10 municipalities including the capital, with a female population of approximately 367,768 women aged 14 to 59 years. This study focused only on the women in this age group, enrolled in public high schools, and the women being treated in basic health units in the municipalities involved, in the period from January 2009 to December 2011.

Our study showed that $87 \%$ of the surveyed women reported having had a preventive Pap test at least once in their lifetime and $69.4 \%$ said they had had the procedure according to the recommended frequency suggested by the Ministry of 
Health of Brazil. This coverage rate of the Pap test is similar to that found by Fernandes et al. [16] in a study involving women from a municipality in the metropolitan region of Natal (64.4\%) and that found in women from Pelotas [17], South Brazil (68.9\%), but below the rates reported in women from São Paulo, Southeastern Brazil [18] (77.3\%). It was also found that $18.0 \%$ of the respondents had not been tested with the recommended frequency, and $12.6 \%$ reported that they had never been tested. These values are smaller when compared with those found in women from a municipality in the metropolitan region of Natal [16], whose percentages were $35.6 \%$ for those who had not been tested with the recommended frequency and $15.0 \%$ for those who had never been tested. The proportion of women who had never had a Pap test in their life, as found by this study, is similar to that found in São Paulo [18] (13.9\%) and in women of Campinas [8] (11.2\%), both located in southeastern Brazil, but is well above that found in women of Medellin, Colombia [7] (7.8\%).

The results show that the surveyed women had a very low knowledge level about the screening test for cervical cancer, since only $47.0 \%$ had a medium or high level of knowledge about the exam, compared with the women from Medellin [7], where this rate reached a value of $97.3 \%$. Our study found a greater proportion of women with a high level of knowledge about the Pap test among those with more education and higher household income. This is probably due to more ease of access available to these women for information on the examination. Our results are concordant in this aspect with those reported for women of Medellin [7]. Also, an association was observed between the middle level of knowledge about the Pap test with marital status and pregnancy, where women married or living in a stable relationship with her partner and those who had at least one pregnancy presented a higher degree of knowledge about the exam. This could be explained by the fact that these women more frequently seek health services for guidance on contraceptive methods or for prenatal exams.

Regarding the knowledge of the women from this study about HPV, although it was very low with only $29.1 \%$ of those interviewed showing a medium or high degree of knowledge on the virus, this proportion is above that described for the women of Medellin [7], but far below that found in women of Sweden [19]. In two other studies conducted in Brazil, involving public school students, one study being performed in the South region [20] and another in the Southeast region [21], very low levels of knowledge about HPV were also found. We observed a greater proportion of women with high-level knowledge about HPV among those aged over 38 years, in those with greater schooling, among the married women or those living in a stable relationship with their partner, in those with higher household incomes, and in those who had had at least one pregnancy. The reason may be that these women have greater ease of access to information on HPV and its role in the development of premalignant and malignant lesions of the genital tract and possibly that these women more frequently seek health services for guidance about contraceptive methods or for prenatal exams. These results are similar to those obtained for women from Medellin [7], regarding the variables of age, education, and socio-economic condition, but differ with respect to marital status. With respect to the association of the high level of knowledge on HPV and the variables marital status and education, our results are also similar to those described for women of Quebec, Canada [22].

Although almost all participants said that they had heard about the Pap test, only a minority could talk about its purpose but, nonetheless, were unaware of the advantages and benefits of having the procedure regularly to prevent cervical cancer. However, a significant proportion $(69.4 \%)$ of the respondents said they had undergone the examination with the frequency recommended by the Health Ministry. This suggests that most of the women had the examination because they were recommended to do so, but they did not know the purpose it served, nor did they possess any degree of awareness about the advantages and benefits of the procedure for their health. On the other hand, a significant portion of respondents had heard about the HPV, and a smaller proportion knew that it is transmitted through sexual contact; however, the majority of them were unable to establish any correlation between genital infection with this virus and the occurrence of cervical lesions, including cancer.

The Brazilian public health system consists of the SUS (Sistema Único de Saúde-Unified Health System), which aims to provide full health coverage to every citizen of Brazil. Actions directed to family planning are inserted within the SUS through the Family Health Program (PSF). However, due to its wide scope, this system has limitations, which sometimes may make access to the health service difficult. Moreover, there is a low degree of knowledge and awareness of the women in relation to HPV and the importance of a periodical Pap test, aimed at the early detection and treatment of cervical lesions associated with HPV and cervical cancer prevention. This is reflected in the low levels of coverage obtained by the cervical cancer screening program. In this context, the findings of this study may serve to arouse the attention of professionals working at PSF about the need to intensify educational activities, aiming to improve the level of knowledge and awareness about the role of HPV in the etiology of uterine cervical lesions, including cervical cancer, and the importance of performing periodic cytologic exams to prevent these diseases.

The present study has some limitations. First, the target population included only women studying in public schools or attending public health units, a population who generally present a lower socioeconomic status and who may not be so representative of the women population of Natal city. Secondly, questions about the HPV vaccine were not included in this research. Although the vaccine is the most effective way to prevent HPV infection, it is not accessible to the majority of the population, since it is not yet effectively included in the Brazilian official program of vaccination.

\section{Conclusion}

Our results show that the majority of women of the segment of the studied population have low levels of knowledge and of conscientization of the role of HPV as a causative agent of precancerous and cancerous lesions of the anogenital tract. 
Furthermore, the majority of women respondents do not have adequate knowledge about the screening test for cervical cancer or about the advantages and benefits of doing so regularly to prevent the disease. Thus, it is clear there is a critical need to improve the knowledge of the local female population on the role of HPV in the etiology of cervical cancer, as well as about the screening method of this disease. Therefore, we suggest educational campaigns emphasizing the role of HPV in the etiology of the cervical lesions of different degrees, including cancer, as well as the importance of regular Pap tests to prevent these diseases.

\section{References}

[1] D. M. Parkin and F. Bray, "Chapter 2: the burden of HPV-related cancers," Vaccine, vol. 24, supplement 3, pp. S11-S25, 2006.

[2] J. Ferlay, H. R. Shin, F. Bray, D. Forman, C. Mathers, and D. M. Parkin, "Estimates of worldwide burden of cancer in 2008: GLOBOCAN 2008," International Journal of Cancer, vol. 127, no. 12, pp. 2893-2917, 2010.

[3] Instituto Nacional de Câncer José Alencar Gomes da Silva, Coordenação Geral de Ações Estratégicas-Coordenação de Prevenção e Vigilância. Estimate/2012-Cancer Incidence in Brazil, 2011, http://www.inca.gov.br/estimativa/2012/.

[4] M. Kyrgiou, G. Valasoulis, C. Founta et al., "Clinical management of HPV-related disease of the lower genital tract," Annals of the New York Academy of Sciences, vol. 1205, pp. 57-68, 2010.

[5] D. Kobayashi, O. Takahashi, C. Hikosaka, T. Okubo, and T. Fukui, "Optimal cervical cytology mass screening interval for cervical cancer," Archives of Gynecology and Obstetrics, vol. 287, no. 3, Article ID 136034, pp. 549-554, 2013.

[6] P. N. Aniebue and U. U. Aniebue, "Awareness and practice of cervical cancer screening among female undergraduate students in a Nigerian University," Journal of Cancer Education, vol. 25, no. 1, pp. 106-108, 2010.

[7] R. Hanisch, J. Gustat, M. E. Hagensee et al., "Knowledge of Pap screening and human papillomavirus among women attending clinics in Medellín, Colombia," International Journal of Gynecological Cancer, vol. 18, no. 5, pp. 1020-1026, 2008.

[8] V. M. S. L. Amorim, M. B. D. A. Barros, C. L. G. César, L. Carandina, and M. Goldbaum, "Factors associated with women's failure to submit to Pap smears: a population-based study in Campinas, São Paulo, Brazil," Cadernos de Saúde Pública, vol. 22, no. 11, pp. 2329-2338, 2006.

[9] S. de Sanjosé, M. Diaz, X. Castellsagué et al., "Worldwide prevalence and genotype distribution of cervical human papillomavirus DNA in women with normal cytology: a metaanalysis," Lancet Infectious Diseases, vol. 7, no. 7, pp. 453-459, 2007.

[10] J. V. Fernandes, R. de Vasconcellos Meissner, M. G. F. de Carvalho, T. A. A. de Medeiros Fernandes, P. R. M. de Azevedo, and L. L. Villa, "Prevalence of HPV infection by cervical cytologic status in Brazil," International Journal of Gynecology and Obstetrics, vol. 105, no. 1, pp. 21-24, 2009.

[11] P. K. S. Chan, A. R. Chang, M. Y. Yu et al., "Age distribution of human papillomavirus infection and cervical neoplasia reflects caveats of cervical screening policies," International Journal of Cancer, vol. 126, no. 1, pp. 297-301, 2010.

[12] X. Castellsagué, "Natural history and epidemiology of HPV infection and cervical cancer," Gynecologic Oncology, vol. 110, no. 3, supplement 2, pp. 4-7, 2008.
[13] F. X. Bosch, S. de Sanjosé, and X. Castellsagué, "HPV and genital cancer: the essential epidemiology," in Vaccines for the Prevention of Cervical Cancer, P. L. Stern and H. C. Kitchener, Eds., chapter 4, Oxford University Press, Oxford, UK, 2008.

[14] M. Stanley, "Pathology and epidemiology of HPV infection in females," Gynecologic Oncology, vol. 117, supplement 2, pp. S5S10, 2010.

[15] M. Arbyn, S. de Sanjosé, M. Soraiya et al., "EUROGIN 2011 roadmap on prevention and treatment of HPV-related disease," International Journal of Cancer, vol. 131, no. 9, pp. 1969-1982, 2012.

[16] J. V. Fernandes, S. H. L. Rodrigues, Y. G. A. S. da Costa et al., "Knowledge, attitudes, and practices related to Pap test in Northeastern Brazil," Revista de Saude Publica, vol. 43, no. 5, pp. 851-858, 2009.

[17] A. A. Hackenhaar, J. A. Cesar, and M. R. Dominuges, "Pap smears of 20-59 year-old women in Pelotas, Southern Brazil: prevalence, approach and factors associated with not undergoing the test," Revista Brasileira de Epidemiologia, vol. 9, no. 1, pp. 103-111, 2006.

[18] A. A. Pinho and I. França Jr., "Cervical cancer prevention: a theoretical framework to analyze Papanicolaou test access and use," Revista Brasileira de Saúde Materno Infantil, vol. 3, no. 1, pp. 95-112, 2003.

[19] L. A. Dahlström, K. Sundstrom, C. Young, C. Lundholm, P. Sparén, and T. N. Tran, "Awareness and knowledge of human papillomavirus in the Swedish adult population," Journal of Adolescent Health, vol. 50, no. 2, pp. 204-206, 2012.

[20] F. S. Conti, S. Bortolin, and I. C. Külkamp, "Education and promotion to the health: behavior and knowledge of adolescents of public and particular college in relation to the human papillomavirus," Jornal Brasileiro de Doenças Sexualmente Transmissíveis, vol. 18, no. 1, pp. 30-35, 2006.

[21] J. R. S. Bretas, C. V. S. Ohara, D. P. Jardim, and R. L. Muroya, "Knowledge of STD/AIDS among adolescent students," Revista da Escola de Enfermagem da USP, vol. 43, no. 3, pp. 551-557, 2009.

[22] H. Cerigo, M. E. Macdonald, E. L. Franco, and P. Brassard, "Awareness and knowledge about human papillomavirus among Inuit women in Nunavik, Quebec," Journal of Community Health, vol. 36, no. 1, pp. 56-62, 2011. 


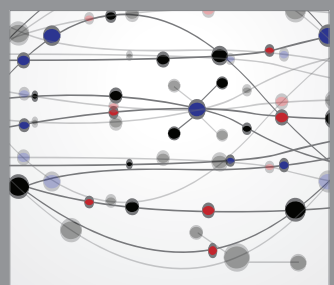

The Scientific World Journal
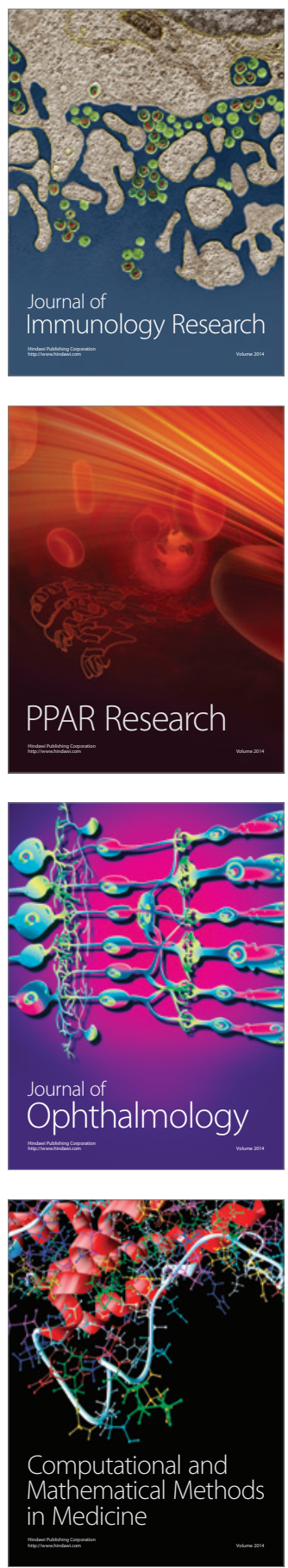

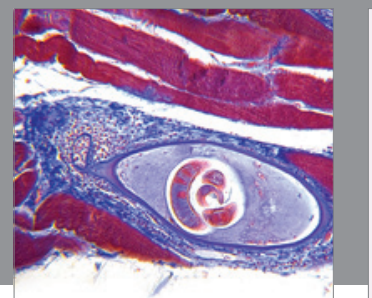

Gastroenterology

Research and Practice
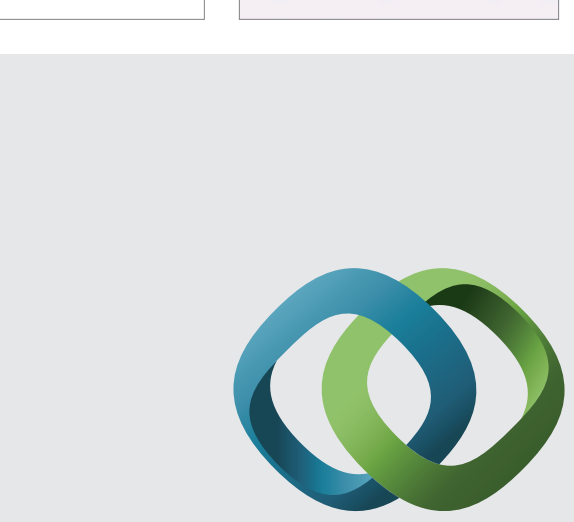

\section{Hindawi}

Submit your manuscripts at

http://www.hindawi.com
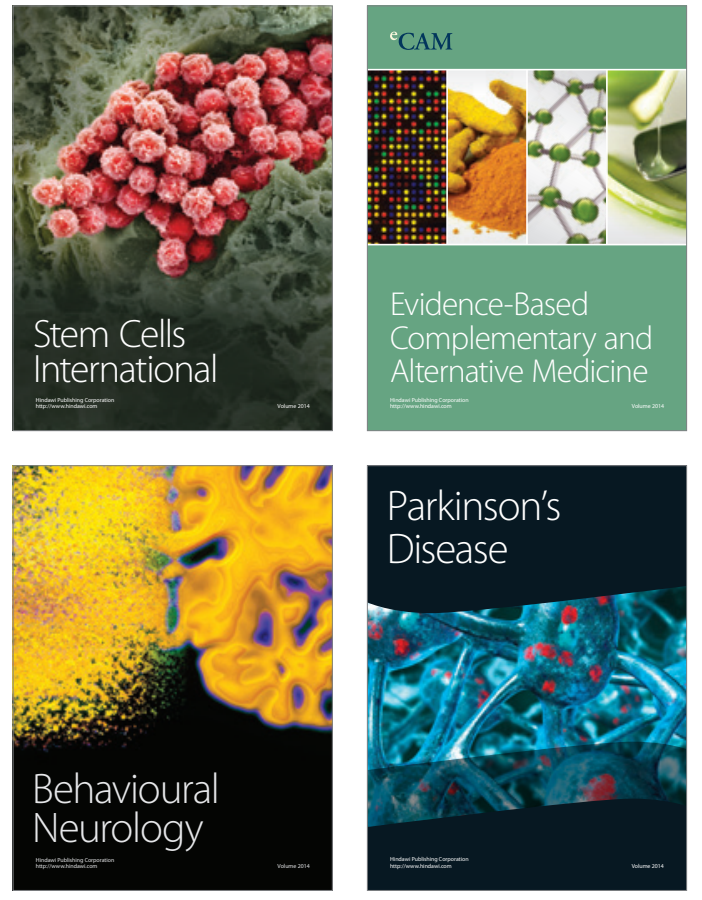
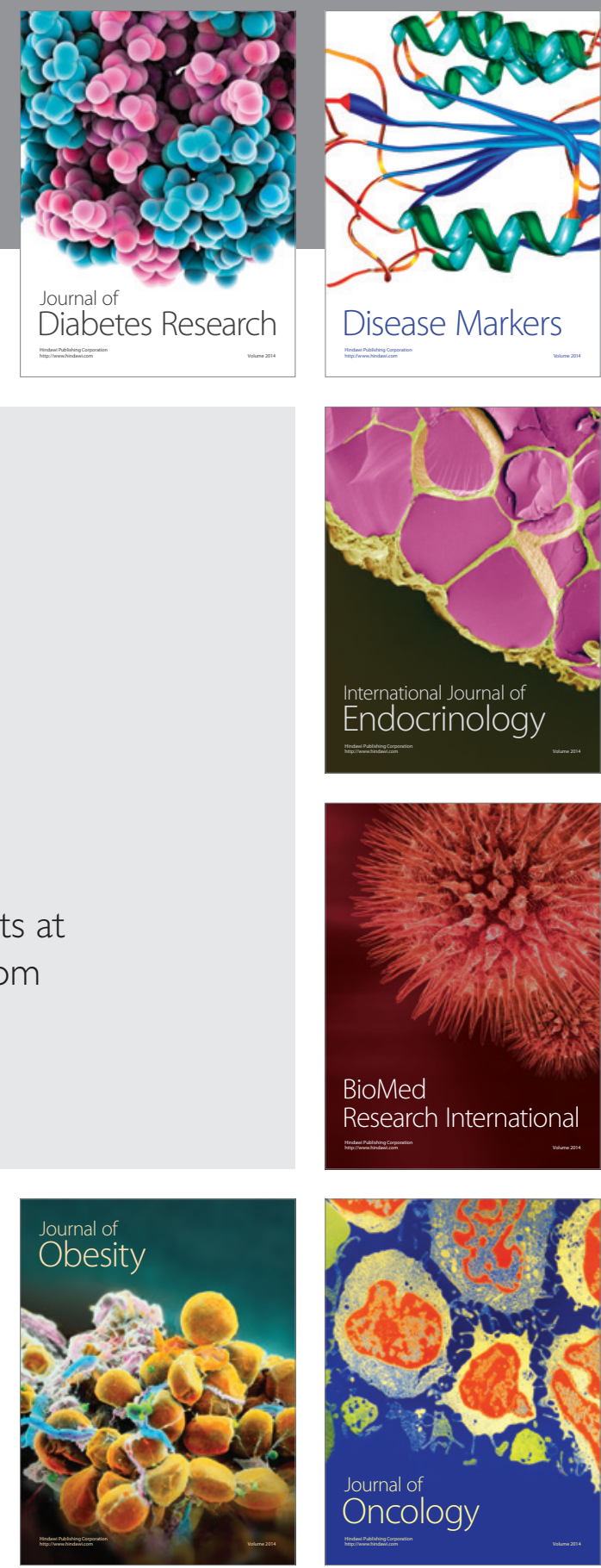

Disease Markers
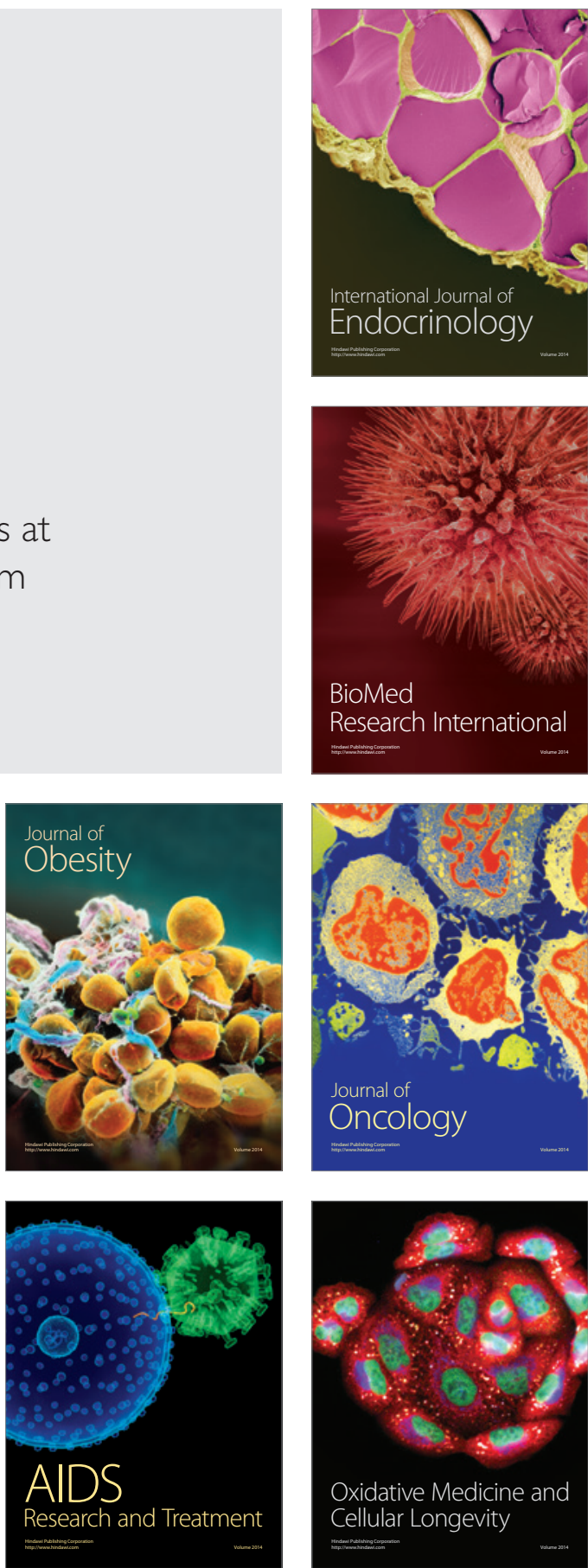